\title{
Fall in the prevalence of chronic gastritis over 15 years: analysis of outpatient series in Finland from 1977, 1985, and 1992
}

\author{
P Sipponen, T Helske, P Järvinen, H Hyvärinen, K Seppälä, M Siurala
}

\begin{abstract}
To investigate whether the occurrence of chronic gastritis (and Helicobacter pylori acquisition) has changed in Finland in the past 15 years, the prevalence rates of chronic gastritis in biopsy specimens in consecutive series of outpatients (aged 20 or more) who had undergone diagnostic upper gastrointestinal endoscopy in 1977 (702 patients), 1985 (1309 patients), or 1992 (1447 patients) were compared. The prevalences of gastritis in these series were also compared with that in a random sample (438 subjects) of people who underwent endoscopy in 1974-76. It seemed that the prevalence rate of gastritis (and of prior $H$ pylori acquisition) had fallen by $18 \%$ on average between 1977 and 1992, and that the prevalence of gastritis was significantly lower in the outpatients in 1992 than in the random endoscopy sample in 1974-76. The reduction was most noticeable in young age groups (20-49 years) in which the decline was $38 \%$ (drop from $66 \%$ to $41 \%$ ). In addition, it seemed that the prevalence of gastritis was very dissimilar in different birth cohorts. The prevalence was high $(70-80 \%)$ in 1977,1985 , and 1992 in the cohorts born at the beginning of the century and lower $(40-50 \%)$ in those born during later decades. The prevalence rates had remained unchanged in the same cohorts over the 15 years (from 1977 to 1992) suggesting that the people had mainly been infected with $H$ pylori and contracted gastritis before the age of 20. In conclusion, gastritis is a cohort phenomenon and its prevalence has fallen in Finland in the last 15 years. This decrease is caused by a decline of the rate of $H$ pylori acquisition in birth cohorts, particularly in childhood and adolescence (below age of 20).

(Gut 1994; 35: 1167-1171)
\end{abstract}

Chronic gastritis is generally an infectious disease. Helicobacter pylori acquisition leads to chronic non-atrophic gastritis, which slowly develops into atrophic gastritis and subsequently to intestinal metaplasia in a significant number of infected subjects. ${ }^{12}$
The most important factor in $H$ pylori positive chronic gastritis is that the gastritis is associated with peptic ulcer disease $\mathrm{e}^{2-4}$ and gastric cancer. ${ }^{2}$ 5-8 These associations are so strong that they may even indicate causality between the disorders. The suggestions of causality presuppose, however, that the epidemiology and time trends of these diseases are alike. Otherwise, assumptions of causal relations would probably be incorrect, and the associations epiphenomena only. The assumption of similar time trends suggests that the prevalence of chronic gastritis (and previous $H$ pylori acquisition) should have fallen in recent decades as is the case in the incidence of gastric cancer ${ }^{9}{ }^{10}$ and peptic ulcer disease. ${ }^{11-14}$

To investigate whether there have been any changes in the occurrence of chronic gastritis in Finland, we compared the prevalence rates of gastritis in biopsy specimens from series of consecutive outpatients who had undergone diagnostic upper gastrointestinal endoscopy in Jorvi Hospital (Espoo, Finland) in 1977, 1985 , or 1992 . In this period, routine random biopsy specimens from both the antrum and corpus were always taken at the diagnostic endoscopy.

\section{Methods}

\section{PATIENT SERIES}

In 1977, 1985, and 1992, all patients who had undergone diagnostic upper gastrointestinal endoscopy in Jorvi Hospital were included in the study. Since 1976, routine random biopsy specimens have always been taken from both antrum and corpus at the first diagnostic endoscopy (on average 1.9 (range 1-4) biopsy specimens from each area), in addition to specimens from all lesions visualised and from the lower duodenum and duodenal bulb. Thus 702, 1309, and 1447 patients from the years 1977, 1985, and 1992 respectively were studied. Patients who had undergone gastric surgery or from whom only specimens taken at operation were available, were excluded. All other patients were included irrespective of the underlying disease.

Jorvi Hospital is a district hospital serving a population of approximately 210000 
inhabitants. The hospital provides a first line ambulatory endoscopy service. Some small private clinics provide a similar service in the same geographical area but are of minor importance with regard to the amount of endoscopy they undertake.

In 1977, 1985, and 1992, the ages of the patients who underwent endoscopy were 50 (15), 52 (16), and 55 (15) years, respectively, and the male to female ratios were $1.0,0 \cdot 8$, and 0.8 respectively. When the patients were classified into six age groups (20-29, 30-39, $40-49,50-59,60-69$ or $70-79$ years), no significant differences (Friedman ANOVA) were found but the proportion of elderly people tended to be higher in 1992 than in 1977.

POPULATION BASED SERIES

The data from the outpatient series were also compared with those from a population based family sample of 438 subjects (age 51 (17) years; $M / F$ ratio 1.0 ) examined endoscopically in 1974-76. This sample of people was originally collected to serve as controls to first degree subjects of gastric cancer patients in other epidemiological studies. ${ }^{15}$ More data on demography and collection of this series are presented elsewhere. ${ }^{15}$

\section{HISTOLOGY}

Endoscopic biopsy specimens were routinely processed and stained with haematoxylin and eosin and Alcian blue ( $\mathrm{pH} 2 \cdot 5$ )-PAS methods. Modified Giemsa was used to stain some (60 cases) of the specimens from 1977 and 1985 to control the specificity of $H$ pylori findings, and routinely in those from 1992. The specimens were consecutively read and reported by the same staff of experienced pathologists according to the principles which correspond to the guidelines of the Sydney System. ${ }^{16}$ In the present study, the patients were classified into three categories as follows: (1) Normal (no evidence of chronic gastritis): normal antrum and corpus; no inflammation, no atrophy or metaplasia. For the sake of simplicity, patients with special forms of gastritis (lymphocytic, eosinophilic, granulomatous, drug-associated gastritis, etc) were included;

(2) Chronic non-atrophic gastritis: chronic gastritis present either in the antrum or corpus, or both; no atrophy;

(3) Chronic atrophic gastritis: atrophy (loss of normal mucosal glands) is present either in antrum or corpus, or both. Intestinal metaplasia was considered an important indicator for atrophy, particularly in antral biopsy specimens. However, the occurrence of mild metaplasia in some single glands (less than $10 \%$ of the volume of the epithelium in available biopsy material) without other evidence of loss of normal glands was not seen to indicate atrophy, and such cases were classified into the category of 'non-atrophic gastritis'.

In the present analysis, patients with atrophy, irrespective of its degree (mild,

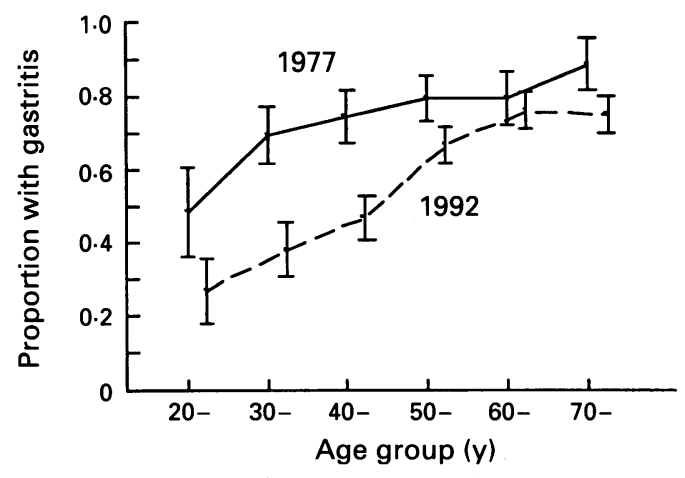

Figure 1: Proportion of patients (men and women) with gastritis (non-atrophic plus atrophic) in 10 year age groups in the outpatient series of 1977 and 1992. The $95 \%$ confidence intervals of the proportions are indicated as vertical bars.

moderate, or severe) were classified into one and the same category - 'chronic atrophic gastritis'. In this study, the terms gastritis or chronic gastritis indicate cases with either chronic 'non-atrophic gastritis' or 'chronic atrophic gastritis'.

\section{REPRODUCTION}

The reproducibility of histological interpretation was examined in random samples of biopsy specimens taken in 1977, 1985, and 1992 by one of the authors (PS). The analysis showed high agreement between the new and former readings. The kappa and lambda values were $0 \cdot 84$ and $0 \cdot 87$, respectively.

\section{H PYLORI}

Tissue specimens were stained routinely (modified Giemsa) for $H$ pylori in 1992 and selected random samples of specimens (60 cases) in 1977 and 1985 were stained for H pylori.

\section{STATISTICS}

Age specific prevalence of gastritis and atrophic gastritis was calculated in six age groups: 20-29, 30-39, 40-49, 50-59, 60-69, 70-79 years, in men and women separately. In calculating statistical significances, we used non-parametric tests $-\chi^{2}$ and non-parametric Friedman ANOVA with concordance. In the calculation of proportions, $95 \%$ confidence intervals $(95 \% \mathrm{CI})$ were also estimated.

\section{Results}

The prevalence of chronic gastritis was lower in all age groups and in both sexes in the outpatient series in 1992 than in 1977 (Fig 1). The fall in prevalence of gastritis in men and women between 1977 and 1992 was $18 \%$ overall and greatest in the three youngest age groups (20-49 years), in which the prevalence had fallen $38 \%$ (from $66 \%$ to $41 \%$ ). The prevalence figures in 1985 were between those in 1977 and 1992 (data not shown). The prevalence of gastritis was lower in all age categories in 1992 than in those of the population based sample of people in 1974-76 (Fig 2). 


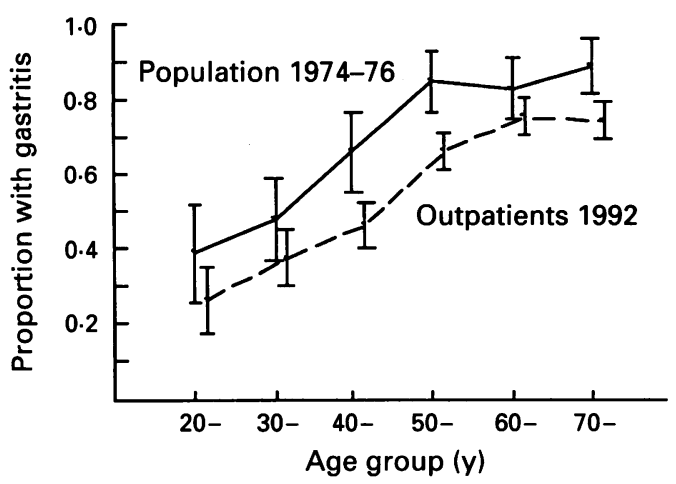

Figure 2: Proportion of subjects (men and women) with chronic gastritis (non-atrophic plus atrophic) in 10 year age groups in the population based family sample of 1974-76 and in the outpatient series of 1992. The 95\% confidence intervals of the proportions are indicated as vertical bars.

Prevalence of atrophic gastritis had also fallen over time, and this was most noticeable in the older age groups (Fig 3).

In all series, the prevalence rate of gastritis correlated positively with age (prevalence increased with age) (Fig 1). No significant differences were found, however, in the prevalences of gastritis in 1977, 1985, and 1992 in the same birth cohorts (Table; the prevalence rates are extrapolated for the birth cohorts from which histological biopsy data for all three time points were available; that is, from 1977, 1985, and 1992). Thus, the prevalence of gastritis had remained quite unchanged in all birth cohorts over the period of 15 years (Table).

In the same categories of non-atrophic and atrophic gastritis, the prevalence of $H$ pylori was similar in all three series. In non-atrophic gastritis, the prevalence of $H$ pylori positive cases (Giemsa stain showing $H$ pylori in either the antrum, corpus, or both) was $94 \%$ versus an average of $50 \%$ in atrophic gastritis (including the cases with autoimmune, atrophic gastritis limited to the gastric body). In atrophic gastritis, the prevalence of $H$ pylori positivity decreased with the increasing degree of atrophy. In addition, in non-atrophic gastritis, few cases with very mild chronic inflammation were negative for $H$ pylori. H heilmannii - (formerly, Gastrospirillum hominis) like organisms could be found in few cases with non-atrophic gastritis.

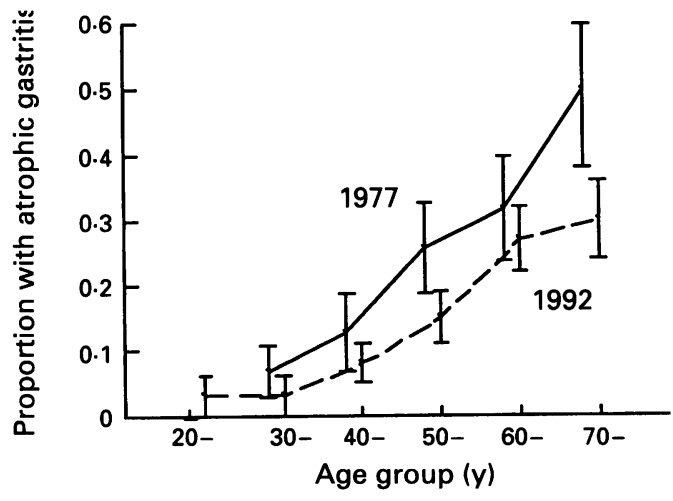

Figure 3: Proportion of patients (men and women) with atrophic gastritis in 10 year age groups in the outpatient series of 1977 and 1992. The $95 \%$ confidence intervals of the proportions are indicated as vertical bars.
Prevalence (\%) of chronic gastritis in four birth cohorts in 1977, 1985, and 1992. The prevalence rates are extrapolated for these birth cohorts (men and women) for which histological biopsy data were available from 1977, 1985, and 1992. The number of cases (10 year age groups) on which the estimate of prevalence is based is given in parentheses

\begin{tabular}{llll}
\hline & \multicolumn{4}{l}{ Prevalence (\%) of chronic gastritis } \\
\cline { 2 - 4 } Cohort born & 1977 & 1985 & 1992 \\
\hline Men: & $50(34)$ & $54(98)$ & $50(114)$ \\
$1948-57$ & $72(67)$ & $65(144)$ & $61(173)$ \\
$1938-47$ & $77(75)$ & $76(130)$ & $77(167)$ \\
$1928-37$ & $80(86)$ & $88(74)$ & $81(101)$ \\
$1918-27$ & $45(31)$ & $44(130)$ & $36(160)$ \\
Women: & $61(61)$ & $59(148)$ & $54(177)$ \\
$1948-57$ & $71(68)$ & $73(130)$ & $66(148)$ \\
$1938-47$ & $77(77)$ & $77(117)$ & $70(159)$ \\
$1928-37$ & & & \\
$1918-27$ & & &
\end{tabular}

\section{Discussion}

The investigation shows that the prevalence rate of chronic gastritis has fallen significantly in the hospital based series in Finland over the past 15 years (from 1977 to 1992). This decrease is found in both non-atrophic gastritis, in both sexes, and in all age groups. In addition, the prevalences of gastritis were lower in the outpatient series in 1992 than in the 1974-76 population based sample.

The decrease has been surprisingly large. From 1977 to 1992 , the drop was $18 \%$ on average, and as much as $38 \%$ in young (20-49 years) age groups (prevalence has decreased from $66 \%$ to $41 \%$ ). Thus, gastritis has become more rare in young people in particular, but still tends to be rather common in the elderly.

Because chronic gastritis is infectious $(H$ pylori $)$ in origin, ${ }^{17} 18$ the reduction in prevalence is probably caused by a decline in the acquisition of $H$ pylori with time - that is, $H$ pylori infections have become more infrequent, particularly in the young generations. Correspondingly, it seems that the persisting ('old') infections have remained relatively common in the older generations.

Comparing the series from 1977 and 1992, it seems that the people showing the same prevalence of gastritis in 1977 and 1992 are 15-20 years older in 1992 than in 1977. Furthermore, extrapolation of the prevalences of gastritis for different birth cohorts in 1977, 1985 , and 1992 shows that these rates have remained practically unchanged in same birth cohorts over the period of 15 years - that is, the prevalence of gastritis has stayed at a high stable level in 'old' cohorts and at a low stable level in 'young' cohorts. For instance, in people (men plus women) born in 1948-57, the prevalences were $48 \%, 48 \%$, and $43 \%$ in 1977, 1985, and 1992, respectively. The corresponding figures were $79 \%, 81 \%$, and $76 \%$ for the birth cohort born in 1918-27.

The observations indicate that every birth cohort has a 'specific' prevalence rate of gastritis, and that these rates vary between the cohorts but remain quite stable over the years in the same cohort. It is likely that it is this cohort-specific rate of gastritis that has fallen in Finland with time.

The conclusions of cohort phenomenon and of infectious origin of gastritis suggest that the 
people in the cohorts tended to be infected and contracted gastritis very early in their life, after which new $H$ pylori infections are quite rare. In the present study, the youngest birth cohort (born in 1948-57) did show a similar prevalence rate of gastritis in 1977, 1985, and 1992, suggesting that the people in the cohort had contracted gastritis before the age 20 . Thus, it seems likely that the infection rate, particularly in childhood or adolescence, decides the prevalence of gastritis for the birth cohort in adulthood. In agreement with these views, the cohort effect in $H$ pylori infections has recently shown in serological studies of Parsonnet et $a l^{20}$ and Banatvala et al, ${ }^{21}$ emphasising that the infection occurs - mainly in childhood.

The cohort phenomenon in gastritis also agrees with other serological studies of $H$ pylori which suggest that the socioeconomic conditions, especially in childhood, are factors that predict the rate of $H$ pylori infection in the population. ${ }^{22-25}$ Poor environmental hygiene in households, absence of a fixed water supply, and overcrowding in childhood are some of the conditions that might increase the risk of $H$ pylori acquisition. ${ }^{22-25}$ Direct serological follow up studies also support these views, and that the acquisition rate is lower in adulthood than we have believed so far. In $H$ pylori seronegative adults, a seroconversion seems to be rather a rare event, the annual infection rate being only $0 \cdot 15-0 \cdot 38 \% .^{26} 27$

The present study indicates that atrophic gastritis has also decreased in prevalence in the past 15 years. This is a logical result. Atrophic gastritis develops over time (years, decades) in some patients with non-atrophic $H$ pylori gastritis. ${ }^{12}$ This process is slow and obviously multifactorial ${ }^{28}$ but results in a loss of normal mucosal glands (atrophy) and the appearance of metaplasia (intestinal and pseudopyloric metaplasia) in the antrum, corpus, or both. A high rate of $H$ pylori infection in childhood would predict a high prevalence of atrophic gastritis and intestinal metaplasia in the cohort (population) in the late adulthood. On the other hand, a decrease in these rates could result in a decrease in the prevalence of atrophic gastritis and metaplasia, as seems to be the case.

Methodological biases can be many, and may easily jeopardise a study of this sort. Indications for endoscopy may have changed in 15 years, thereby causing selection biases. This may also be the case regarding the criteria and techniques used in endoscopy and histological examinations. However, no significant differences occurred in the demographic data of patients in 1977, 1985, and 1992, suggesting that the selection biases, even though existing, may not play the major role. In addition, the prevalence figures on gastritis were significantly lower in the outpatient series in 1992 than in the random sample of people in 1974-76, supporting the view that the present observations are true and not caused by biases in the selection only. Had the decrease in prevalence of gastritis been caused by changes in the inclusion criteria, it would be logical to assume that gastritis should be more common in the outpatient series than in the random sample of people. This was not the case.

In addition, to collect data for epidemiological studies, it was initially planned, and achieved from 1976, that routine histological biopsy specimens should be collected from the same areas (antrum and corpus) of the stomach in every patient undergoing diagnostic endoscopy. The histology was also graded by the same staff of pathologists according to principles corresponding to the Sydney System. These interpretations seem to have been consistent over the period of 15 years. Kappa and lambda coefficients were high, indicating good concordance.

In conclusion, the study indicates that the prevalence of chronic gastritis has fallen significantly in Finland in the past 15 years. This decrease is obviously based on a decrease in the rate of $H$ pylori acquisition with time, particularly in childhood and adolescence (age below 20). Observations on time trends suggest that gastritis is a cohort phenomenon in the population: every birth cohort has a 'specific' prevalence rate that remains unchanged for years (for the whole life), and the rate of $H$ pylori acquisition in childhood and adolescence decides this prevalence rate for the cohort. It seems that the "cohort specific' prevalence of $H$ pylori gastritis is high $(70-80 \%)$ in generations born at the beginning of the century but lower $(40-50 \%)$ in those born in the last decades.

This paper is dedicated to Professor Max Siurala on the occasion of his 80th birthday in October 1993.

1 Siurala M, Sipponen P, Kekki M. Chronic gastritis: dynamic and clinical aspects. Scand $\mathcal{f}$ Gastroenterol 1985; 20 (suppl 109): 69-76.

2 Sipponen P, Kekki M, Siurala M. The Sydney system: epidemiology and natural history of chronic gastritis. Gastroenterol Hepatol 1991; 6: 244-51.

3 Goodwin CS, Armstrong JA, Marshall BJ. Campylobacter pyloridis, gastritis and peptic ulceration. F Clin Pathol 1986; 39: 353-65

4 Graham DY. Helicobacter pylori: Its epidemiology and its role in duodenal ulcer disease. $\mathcal{F}$ Gastroenterol Hepatol 1991; 6: 105-13.

5 Forman D, Newell DG, Fullerton F, Yarnell JW, Stacey $\mathrm{AR}$, Wald $\mathrm{N}$, et al. Association between infection with Helicobacter pylori and risk of gastric cancer: evidence from a prospective investigation. BMF 1991; 302: 1302-5.

6 Parsonnet J, Friedman GD, Vandersteen DP, Chang Y, Vogelman JH, Orentreich N, et al. Helicobacter pylori Vogelman JH, Orentreich $\mathrm{N}$, et al. Helicobacter pylori
infection and risk of gastric cancer. $N$ Engl $\mathcal{f}$ Med 1991; 325: $1127-31$.

7 Nomura A, Stemmermann GN, Chyou P-H, Kato I, PerezPerez GI, Blaser MJ. Helicobacter pylori infection and gastric carcinoma among Japanese Americans in Hawaii. N Engl F Med 1991; 325: 1132-6.

8 Sipponen P, Kosunen TU, Valle J, Riihelä M, Seppälä K. Helicobacter pylori infection and chronic gastritis in gastric cancer. F Clin Pathol 1992; 45: 319-23.

9 Muir C, Waterhouse J, Mack T, Powell J, Whelan S. Cancer incidence in five continents. Vol V. Lyon: IARC, 1987.

10 Howson CP, Hiyama T, Wynder EL. The decline in gastric cancer: epidemiology of an unplanned triumph. Epidemiol Rev 1986; 8: 1-27.

11 Gustavsson $S$, Nyren $O$. Time trends in peptic ulcer surgery, 1956 to 1986 . A nation-wide survey in Sweden. Ann Surg 1989; 210: 704-9.

12 Sonnenberg A. Changes in physician visits for gastric and duodenal ulcer in the United States during 1958-84 as shown by National Disease and Therapeutic Index shown by National Disease and
(NDTI). Dig Dis Sci 1987; 32: 1-7.

13 Bloom BS. Cross-sectional changes in the effects of peptic ulcer disease. Ann Intern Med 1991; 114: 558-62.

14 Kurata JH, Haile BM. Epidemiology of peptic ulcer disease. Clin Gastroenterol 1984; 13: 289-307.

15 Ihamäki T, Varis K, Siurala M. Morphological, functional and immunological state of the gastric mucosa in gastric carcinoma families. Comparison with a computermatched family sample. Scand $\mathcal{f}$ Gastroenterol 1979; 14: 801-12. 
16 Price AB. The Sydney system: histological division. f Gastroenterol Hepatol 1991; 6: 209-22.

17 Marshall BJ, Warren JR. Unidentified curved bacilli in the stomach of patients with gastritis and peptic ulceration. Lancet 1984; i: 1311-15.

18 Lee A. Helicobacter pylori: Causal agent in the peptic ulcer. Microbiological aspects. $\mathcal{f}$ Gastroenterol hepatol 1991; 6: Microbiological aspects. F Gastroenterol hepatol 1991; 6: 19 Blaser

Blaser MJ. Hypothesis on the pathogenesis and natural history of Helicobacter pylori-induced inflammation. Gastroenterology 1992; 102: 720-7.

20 Parsonnet J, Blaser MJ, Perez-Perez GI, Hargrett B, Tauxe RV. Symptoms and risk factors for Helicobacter pylori infection in a cohort of epidemiologists. Gastroenterology 1992; 102: 41-6.

21 Banatvala N, Mayo K, Megraud F, Jennings R, Decks JJ, Feldman RA. The cohort effect and Helicobacter pylori. F Infect Dis 1993; 168: 219-21.

22 Mendall MA, Goggin PM, Molineaux N, Levy J, Toosy T, Strachan D, Norfield TC. Childhood living conditions and Helicobacter pylori seropositivity in adult life. Lancet 1992; 339: 896-7.
23 Jones DM, Eldridge J, Whorwell PJ. Antibodies to Campylobacter pyloridis in household contacts of infected people. BMF 1987; 294: 615.

24 Drumm B, Perez-Perez G, Blaser MJ, Sherman PM. Intrafamilial clustering of Helicobacter pylori infection. N Engl f Med 1990; 322: 359-63.

25 Hazell SL, Mitchell HM, HU PJ, Li YY, Wang ZJ, Shao SM, Liu Q, Chen M, Du GG, Lee A. Gastric cancer - is childhood acquisition of $\mathrm{H}$ pylori the key? Irish $\mathcal{F} M e d ~ S c i$ 1992; 161: 22.

26 Kuipers EJ, Pena AS, Kamp van G, Uyterlinde AM, Pals G, Pels NF. Kurz-Pohlman E, Meuwissen SG. Serocols NF. Kurz-Pohlman E, Meuwissen SG. Seroconversion

27 Cullen DJ, Collins BJ, Cullen KJ, Christianssen K, Epis J, Surveyor I, Warren JR. A 21 year follow-up of Helicobacter pylori infection - the cohort theory proven. Gut 1993; 33: S4.

28 Correa P. Chronic gastritis. In: Gastrointestinal and oesophageal pathology. Whitehead $\mathrm{R}$, ed. London: oesophageal pathology. Whitehead
Churchill Livingstone, 1989; 402-20. 